\title{
NONIMMERSIONS OF LOW DIMENSIONAL FLAG MANIFOLDS
}

\author{
FRED J. CONNELL ${ }^{1}$
}

\begin{abstract}
Certain useful quadratic identities in the cohomology of classifying spaces induce quadratic equations in the cohomology of a manifold $M$ under the classifying map for the normal bundle of $M$. In low dimensional flag manifolds, one can show that the quadratic equation has no root, thus establishing a nonimmersion.
\end{abstract}

1. Introduction. Let $B S O(r)$ be the classifying space for oriented real $r$-plane bundles. In integral cohomology, one has the quadratic identity

$$
p_{r}=\chi^{2} \in H^{4 r}(B S O(2 r) ; Z),
$$

where $p_{r}$ is the $r$ th Pontryagin class and $\chi$ is the Euler class. By a wellknown result of Hirsch [6], an oriented manifold $M$ immerses in codimension $k$ iff the stable normal bundle $v$ has dimension $k$; i.e., $v$ is classified by a map $f_{v}: M \rightarrow B S O(k)$. Thus, if $k$ is even, identity (1.1) induces a quadratic equation in $H^{*}(M ; Z)$. One obtains nonimmersion by showing that this equation has no root.

We shall restrict our attention to complex flag manifolds,

$$
F\left(n_{1}, n_{2}, \cdots, n_{k}\right)=U(n) / U\left(n_{1}\right) \times U\left(n_{2}\right) \times \cdots \times U\left(n_{k}\right),
$$

where $n=n_{1}+n_{2}+\cdots+n_{k}$. We shall show that

(a) If $M=F(2,2)$, then $\operatorname{dim} M=8, M \subseteq R^{14}, \ddagger R^{12}$.

(b) If $M=F(2,3)$, then $\operatorname{dim} M=12, M \subseteq R^{23}, \ddagger R^{19}$.

The methods of this paper also show that

(c) If $M=F(1,2,2)$, then $\operatorname{dim} M=16, M \subseteq R^{22}, \$ R^{20}$.

(d) If $M=F(2,2,2)$, then $\operatorname{dim} M=24, M \subseteq R^{33}, \nsubseteq R^{30}$.

The methods of this paper were first used by Feder [5] to obtain nonimmersions of projective spaces $C P^{n}, n$ odd. Mayer [9] obtains very general

Received by the editors June 6, 1973.

AMS (MOS) subject classifications (1970). Primary 57A35, 57F15; Secondary 55B15, $55 \mathrm{~F} 40$.

Key words and phrases. Normal bundle, mixing construction, Chern class, Pontryagin class, Euler class, classifying space.

${ }^{1}$ The author was supported by a National Science Foundation Graduate Fellowship during the preparation of part of this work.

(f) American Mathematical Society 1974 
nonimmersion results for manifolds, but our methods give stronger results for the flag manifolds considered here.

2. The adjoint representation. The normal bundle of any homogeneous space, and in particular, of flag manifolds, is determined by the adjoint representation. Let $G$ be a Lie group and $H$ a closed subgroup. Let $\pi$ denote the $H$-bundle $H \rightarrow G \stackrel{\pi}{\rightarrow} G / H$ where $G \rightarrow G / H$ is the canonical projection onto the space of right cosets of $H$ in $G$. The space $G / H$ is called a homogeneous space. The mixing construction $\alpha_{\pi}: R O(H) \rightarrow K O(G / H)$ from real representation theory to $K$-theory is a ring morphism defined by letting $\alpha_{\pi}[\rho] \in K O(G / H)$ be the bundle

$$
G / H \stackrel{f_{\pi}}{\longrightarrow} B H \stackrel{B_{\rho}}{\longrightarrow} B O(n),
$$

where $f_{\bar{\pi}}$ classifies the bundle $\pi$ over $G / H$, and $\rho: H \rightarrow O(n)$ is a representation.

Borel and Hirzebruch [4, (1.1)] have determined the tangent bundle $\tau_{G / H}$ of any homogeneous space $G / H$ to be

$$
\tau_{G / H}=\alpha_{\pi}\left(\operatorname{Ad}_{G} \mid H-\operatorname{Ad}_{H}\right) \in K O(G / H) .
$$

Here $\operatorname{Ad}_{H}$ is the adjoint representation of $H$ and $\operatorname{Ad}_{G} \mid H$ is the restriction of $\operatorname{Ad}_{G}$ to $H$. Note that by $(2.1), \alpha_{\pi}\left(\operatorname{Ad}_{G} \mid H\right)$ is the bundle

$$
G / H \stackrel{f_{\pi}}{\longrightarrow} B H \stackrel{B i}{\longrightarrow} B G \stackrel{B A d}{\longrightarrow} B O(g),
$$

where $i: H \rightarrow G$ is the inclusion and $g=\operatorname{dim} G$. Since $B i \circ f_{\pi}$ is homotopically trivial, $\alpha_{\pi}\left(\operatorname{Ad}_{G} \mid H\right)=g$. Hence, we have

$$
\tau_{G / H}=g-\alpha_{\pi}\left(\mathrm{Ad}_{H}\right) .
$$

Thus, the normal bundle $v_{G / H}=\operatorname{dim} G / H-\tau_{G / H}$ is given by

$$
v_{G / H}=\alpha_{\pi}\left(\operatorname{Ad}_{H}\right)-h,
$$

where $h=\operatorname{dim} H$.

3. Cohomology of the flag manifolds. In this section, we compute both the integral cohomology and complex $K$-theory cohomology of the flag manifolds. We adopt the following notation. Let $G=U(n), H=U\left(n_{1}\right) \times$ $\cdots \times U\left(n_{k}\right)$, thus the flag manifold $F=F\left(n_{1}, \cdots, n_{k}\right)$ is given by $F=G / H$. Denote by $w: H \rightarrow G$ the usual inclusion map given by direct sum of matrices, and let $f_{\pi}: F=G / H \rightarrow B H$ be the classifying map for the principal $H$-bundle $H \rightarrow G \stackrel{\pi}{\rightarrow} F=G / H$.

Let $i: p \rightarrow X$ be the inclusion of a point, and define $\bar{H}(X)=\operatorname{Ker} i^{*}$ : $H^{*}(X) \rightarrow H^{*}(p)$. Then we have the following result of Borel [3]. 
Proposition 3.1. The integral cohomology of $F=F\left(n_{1}, \cdots, n_{k}\right)$ is torsion-free and is determined by the following short exact sequence of rings: $O \longrightarrow \bar{H}(B G ; Z) \cdot H^{*}(B H ; Z) \longrightarrow H^{*}(B H ; Z) \stackrel{f_{\pi}^{*}}{\longrightarrow} H^{*}(F ; Z) \longrightarrow 0$.

Here, the term $\bar{H}(B G) \cdot H^{*}(B H)$ is the ideal of $H^{*}(B H)$ generated by $\bar{H}(B G)$.

We explicitly compute generators and relations for the cohomology of $F(2,2)=U(4) / U(2) \times U(2)$. If $p(X, t)$ denotes the Poincaré power series $p(x, t)=\sum_{i \geqq 0} \operatorname{rank} H^{i}(X ; Z) t^{i}$ for any space $X$, then by [8], we have

$$
\begin{aligned}
p(F(2,2), t) & =p(B U(2) \times B U(2), t) / p(B U(4), t) \\
& =1+t^{2}+2 t^{4}+t^{6}+t^{8} .
\end{aligned}
$$

If we now define $x_{i} \in H^{2 i}(F(2,2) ; Z), i=1,2$, by $x_{i}=f_{\pi}^{*}\left(a_{i}\right)$, where $a_{i}=$ $c_{i} \otimes 1 \in H^{*}(B U(2) ; Z) \otimes H^{*}(B U(2) ; Z)$ is the Chern class of the first factor in $B U(2) \times B U(2)$ and $f_{\pi}: F(2,2) \rightarrow B U(2) \times B U(2)$ the usual map, we have

Proposition 3.3. The cohomology ring $H^{*}(F(2,2) ; Z)$ is torsion-free of rank 6 with additive generators $1 \in H^{0}, x_{1} \in H^{2}, x_{1}^{2}$ and $x_{2} \in H^{4}, x_{1} x_{2} \in H^{6}$, and $x_{1}^{2} x_{2} \in H^{8}$.

Remark. We have, for example, $x_{1}^{3}=2 x_{1} x_{2}$.

Proof. From (3.1) and (3.2), we know that $H^{*}(F(2,2), Z)$ is torsionfree of rank 6 . The six elements listed additively generate the image of $f_{\pi}^{*}: H^{*}(B U(2) \times B U(2)) \Rightarrow H^{*}(F(2,2))$. Since $f_{\pi}^{*}$ is onto by $(3.1)$, we are done.

Letting $\alpha_{i}=\gamma_{i} \otimes 1 \in R U(2) \otimes R U(2), i=1,2$ where $\gamma_{i}$ is the $K$-theory Chern class (see $[1, \S 2]$ ), we define $\xi_{i}=\alpha_{\pi}\left(\alpha_{i}\right) \in K F(2,2), i=1$, 2, where $\alpha_{\pi}: R U(2) \times R U(2) \rightarrow K F(2,2)$ is the mixing construction.

PROPOSITION 3.4. The K-theory ring $K F(2,2)$ is torsion-free of rank 6 with additive generators $1, \xi_{1}, \xi_{1}^{2}, \xi_{2}, \xi_{1} \xi_{2}$, and $\xi_{1}^{2} \xi_{2}$. Furthermore, the ring structure of $K F(2,2)$ is formally the same as that of $H^{*}(F(2,2) ; Z)$.

Proof. The Chern character ch: $K F \rightarrow H^{* *}(F ; C)$ is calculated to be

$$
\begin{aligned}
& \operatorname{ch}\left(\xi_{1}\right)=x_{1}+\frac{1}{2}\left(x_{1}^{2}-2 x_{2}\right)-\frac{1}{6} x_{1} x_{2}, \\
& \operatorname{ch}\left(\xi_{2}\right)=x_{2}+\frac{1}{2} x_{1} x_{2}+\frac{1}{1 \frac{1}{2}} x_{1}^{2} x_{2},
\end{aligned}
$$

where the $x_{i}$ are the generators of (3.3). The proof now follows immediately from $[2, \S 2.5]$.

4. The quadratic equation. We now apply identity (1.1) to obtain nonimmersion of $F(2,2)=U(4) / U(2) \times U(2)$ in codimension 4 . If $F(2,2)$ immerses in codimension 4, its stable normal bundle $v$ is an $S O(4)$ bundle. 
Letting $r=2$, identity (1.1) induces a quadratic equation $p_{2}(v)=\chi^{2} \in$ $H^{8}(F(2,2) ; Z)$ for some element $\chi$. Using $(2.2)$, one calculates $p_{2}(v)=$ $-6 x_{1}^{2} x_{2}$, where $x_{1}^{2} x_{2}$ is the generator of $H^{8}$ given in (3.3). Solving for $\chi$, we write $\chi=a x_{2}+b x_{1}^{2} \in H^{4}$ and obtain $-6=(a+b)^{2}+b^{2}$. There are no integer solutions, and thus nonimmersion in codimension 4 is established. Tornehave [10] has established positive immersion results for flag manifolds. Thus, we have

Proposition 4.1. The eight dimensional manifold $F(2,2)$ does not immerse in codimension 4 but does immerse in codimension 6.

The $K$-theoretic methods of [1] and [5] give no new results for $F(2,2)$.

5. Another quadratic equation. Turning attention to the manifold $F(2,3)$, we recall that $\operatorname{Spin}(k)$ is the double cover of $S O(k)$, and that $\operatorname{Spin}^{c}(k)$ is defined by the following pullback diagram:

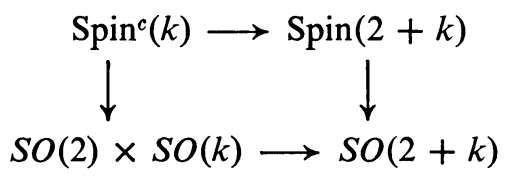

Here, $S O(2) \times S O(k) \rightarrow S O(2+k)$ is the standard inclusion.

For any group $G$, let $R G$ denote the ring of unitary representations. Following [7], we define an element $\tilde{\Delta} \in R \operatorname{Spin}^{c}(2 k+\varepsilon), \varepsilon=0$ or 1 , by $\tilde{\Delta}=z_{0}^{1 / 2} \prod_{i=1}^{k}\left(z_{i}^{1 / 2}+z_{i}^{-1 / 2}\right)$, where $z_{0}$ is the basic toral representation of $S O(2)$ and $z_{i}, i=1, \cdots, k$, are the toral representations of $S O(2 k+\varepsilon)$. One calculates that $\tilde{\Delta}$ satisfies

$$
a_{\varepsilon} \tilde{\Delta}^{2}=z_{0} \cdot \lambda_{1} \in R \operatorname{Spin}^{c}(2 k+\varepsilon), \quad \varepsilon=0 \text { or } 1,
$$

where $a_{0}=1, a_{1}=2$ and $\lambda_{1}$ is the sum of the exterior power elements $\lambda^{i}=$ $\sigma_{i}\left(z_{1}, z_{1}^{-1}, \cdots, z_{k}, z_{k}^{-1},(1)\right), \quad i=0,1, \cdots, 2 k+\varepsilon, \quad \sigma_{i}=i$ th $\quad$ symmetric function.

Since the third integral Stiefel-Whitney class $W_{3}$ of $F(2,3)$ is zero, we know [7, (5.5)] that $F(2,3)$ is a Spin ${ }^{c}$ manifold. In fact, by an appropriate choice of an $S O(2)$ bundle $\delta$ over $F(2,3)$, one can factor the normal bundle $F(2,3) \stackrel{v}{\rightarrow} B S O(n)$ as follows:

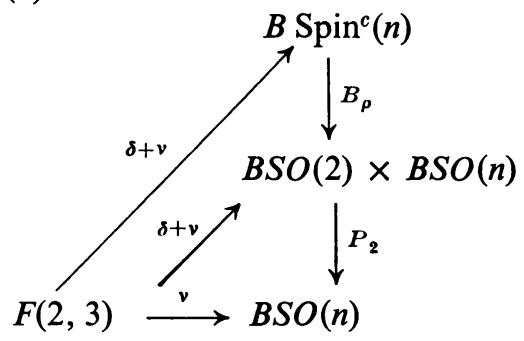

where $\rho$ is the double cover and $p_{2}$ is projection on the second factor. 
If now, $F(2,3)$ immerses, in codimension 7 , the above diagram gives a mapping $F(2,3) \stackrel{\delta+v}{\longrightarrow} B \operatorname{Spin}^{c}(7)$ factoring the normal bundle $F(2,3) \stackrel{v}{\rightarrow}$ $B S O(7)$. Thus identity (5.1) induces a quadratic equation in $K F(2,3)$. One calculates $K F(2,3)$ as in (3.4), and shows that the coefficient of the $\xi_{1}^{2} \xi_{2}$ term in $\tilde{J}$ is $7 / 2$. Thus, there is no integral solution for $\tilde{\Delta}$, and nonimmersion in codimension 7 is established. A positive immersion result for $F(2,3)$ follows from [10]. Thus, we have

Proposition 5.2. The 12-dimensional manifold $F(2,3)$ does not immerse in codimension 7, but does immerse in codimension 11.

\section{BIBLIOGRAPHY}

1. M. F. Atiyah, Immersions and embeddings of manifolds, Topology 1 (1962), 125132. MR $26 \# 3080$.

2. M. F. Atiyah and F. Hirzebruch, Vector bundles and homogeneous spaces, Proc. Sympos. Pure Math., vol. 3, Amer. Math. Soc., Providence, R.I., 1961, pp. 7-38. MR 25 \#2617.

3. A. Borel, Sur la cohomologie des espaces fibrés principaux et des espaces homogènes de groupes de Lie compacts, Ann. of Math. (2) 57 (1953), 115-207. MR 14, 490.

4. A. Borel and F. Hirzebruch, Characteristic classes and homogeneous spaces. I, Amer. J. Math. 80 (1958), 458-538. MR 21 \#1586.

5. S. Feder, Non-immersion theorems for complex and quaternionic projective spaces, Bl. Soc. Mat. Mexicana (2) 11 (1966), 62-67. MR 38 \#721.

6. M. W. Hirsch, Immersions of manifolds, Trans. Amer. Math. Soc. 93 (1959), 242-276. MR 22 \#9980.

7. F. Hirzebruch, A Riemann-Roch theorem for differentiable manifolds, Paris Univ., Séminaire Bourbaki, 1 lième année; 1958/59, Exposés 177, 1959. MR 28 \#1091.

8. A. Liulevicius, Characteristic classes and cobordism. Part I, Aarhus Univ., Lecture Notes, Fall 1967.

9. K. H. Mayer, Elliptische differentialoperatoren und ganzzahligkeitsätze für charakteristische Zahlen, Topology 4 (1965), 295-313. MR 33 \#6650.

10. J. Tornehave, Immersions of complex flagmanifolds, Math. Scand. 23 (1968), 22-26. MR 40 \#4970.

Department of Mathematics, Millikin University, Decatur, Illinois 62522 